\title{
An Innovative Construction of Wheelchair for Handicapped Persons
}

\author{
Samsul Islam Shawon ${ }^{1}$, Mohammad Majidul Haque Bhuiyan ${ }^{2}$, Tazdik Patwary Plateau ${ }^{3}$ \\ ${ }^{1}$ Department of Electrical and Electronics Engineering, American International University, Dhaka, Bangladesh \\ ${ }^{2}$ Department of Electrical and Computer Science Engineering, North South University, Dhaka, Bangladesh \\ ${ }^{3}$ Department of Materials and Metallurgical Engineering, Bangladesh University of Engineering and Technology, Dhaka, Bangladesh
}

Email address:

samsulislamshawon@yahoo.com (S. I. Shawon), majidul.haque@northsouth.edu (M. M. H. Bhuiyan), ptazdik@gmail.com (T. P. Plateau)

\section{To cite this article:}

Samsul Islam Shawon, Mohammad Majidul Haque Bhuiyan, Tazdik Patwary Plateau. An Innovative Construction of Wheelchair for Handicapped Persons. International Journal of Science and Qualitative Analysis. Vol. 4, No. 1, 2018, pp. 13-19.

doi: $10.11648 /$ j.ijsqa.20180401.13

Received: December 19, 2017; Accepted: January 23, 2018; Published: February 5, 2018

\begin{abstract}
This paper represents an important issue with accumulating separate fundamental mechanisms through the wheelchair for the handicapped persons as world is getting tired by seeing the handicapped scenarios for accidental or natural circumstances. Our job is to provide them a pretty easier life with the invention of this modified wheelchair. This chair consists of some important section of project for the handicapped person where all the belongings have been powered by mainly Arduino Uno. Along with this some additional devices like sensor, microcontroller, Bluetooth module, buzzed module and Android software have been used to fulfil the requirement. The chair moves with the directions of the navigation keys from Android mobile. For the deaf person a display has been used with a view to making better communication level with others by the voice command from Android mobile software. Besides, it can detect the obstacle from the front position for better justification of the user. However, this chair is sensible in detecting the hole or crack from the front position of the chair. Sensing living being from the front position is also a responsible duty which will be helpful for blind person indeed. Not only this but also a strong automation system has been provided to control the home appliances with the help of Android software. A 12.5 volt $1 \mathrm{Amp}$ and 5V Battery have been used to power the tasks. This intelligent chair can carry up to 150 kilogram weight which will make a revolution indeed. The entire cost for this job was TK 13500 BDT (150 USD).
\end{abstract}

Keywords: Bluetooth, DC Motors, Home Automation, Sonar Detection, Relays

\section{Introduction}

Today's world has become more and more comfortable for people. In a target to do something for the disabled person, this research team conjunct of three universities made an innovative wheelchair project. The project work has the capability in making the life of handicapped persons quite easier throughout the world. The mechanism access to make the entire project perfectly by accumulation of different codes and project along with the fundamental methodology of particular equipment is not very difficult to acquire. The Arduino Uno simulated code reach with $5 \mathrm{~V}$ from the source to meet with connection of movement of wheelchair [1-8].

Relay is activated to run two motors with the mechanism of the code powered by the $12.53 \mathrm{~V} 1 \mathrm{Amp}$ battery. Apart from this, the $12 \times 6$ display is activated through arduino to meet with voice command via app [9-13]. In another research, solar cells are using to generate energy and also a storage system of Lithium Ion battery was used. An automated robotic system can be chosen for the development of this newest type of wheelchairs [14-19]. Here, Arduino activate the Bluetooth $\mathrm{HC}-05$ for receiving the voice as character for the display and make the communication as well. For sensing the obstacle and crack, Sonar, IR, PIR sensors have been used to indicate the distance, build a strong signal for hole and living being as well. All of them are powered by Arduino except crack sensing part with particular codes. The home automation system has been developed with the Arduino also with less sophisticated mechanism [20-23]. 


\section{Block Diagram}

up the wheelchair and so the most updated equipment and

This research team have tried our utmost to gradually build system to the arrangement were added.

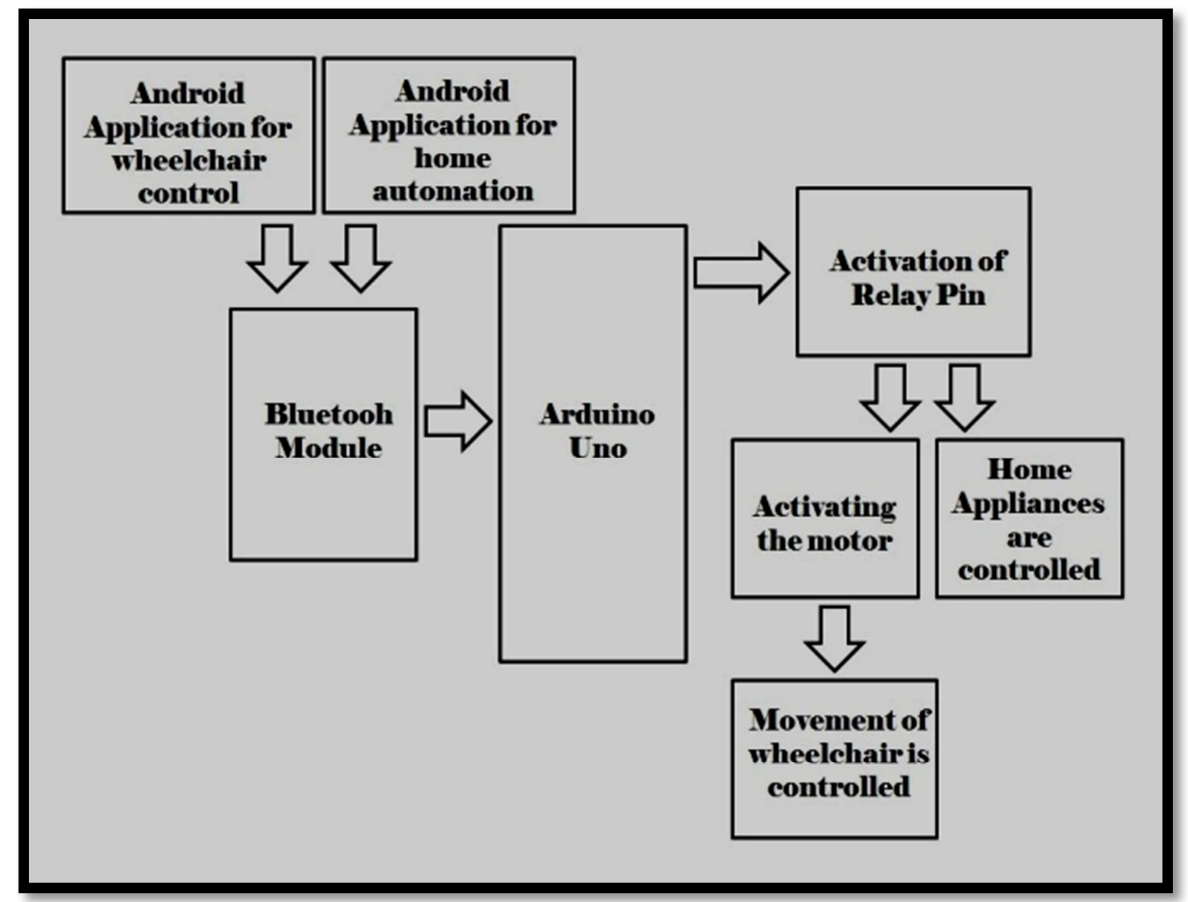

Figure 1. Block diagram of accumulated features of Android control wheelchair movement and home automation.

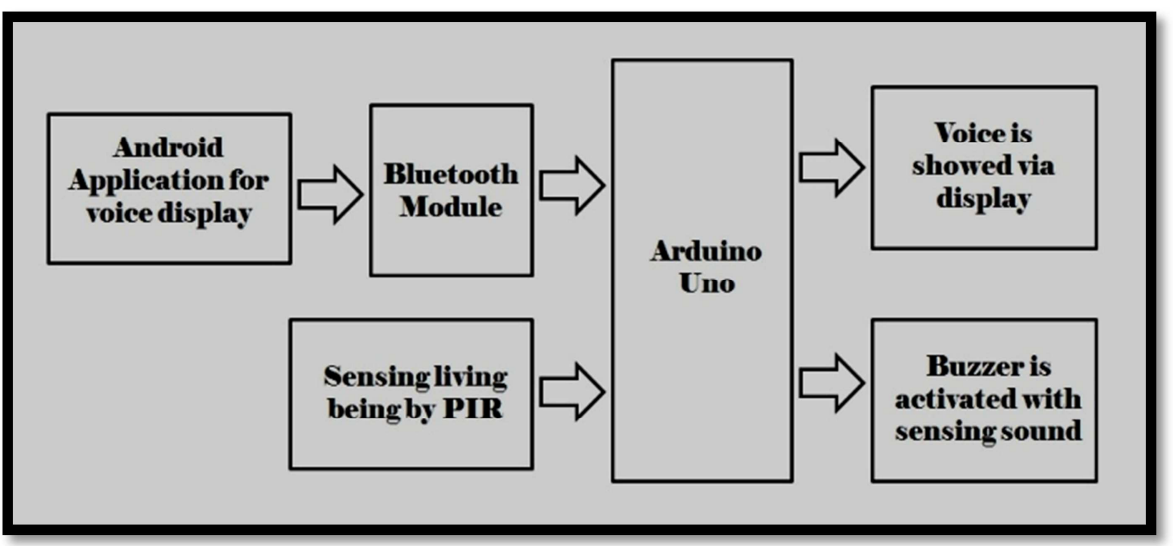

Figure 2. Block diagram of accumulated features of Android control voice to display and Living being sensing PIR.

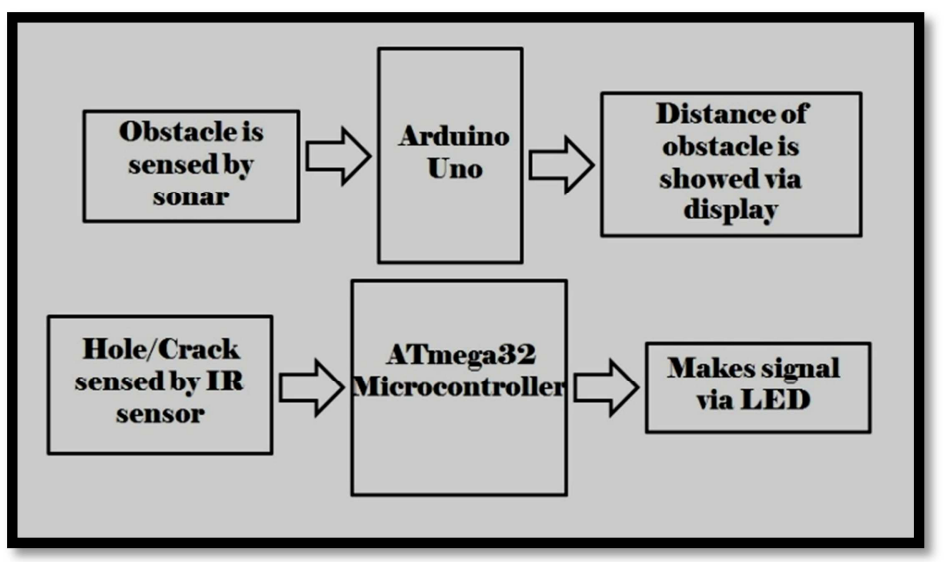

Figure 3. Block diagram of obstacle sensing using sonar (top) and crack sensing using IR (bottom). 


\subsection{Theory and Methodology}

Using the input voltage from the Arduino board (VIN) as an external power source, android control based movement of wheelchair is accomplished. It powered the components and other belongings. It is supplied by USB or another regulated 5V supply. A 3.3 volt supply generated by the onboard regulator carries out the power supply finally. The signal generated from Android mobile is transmitted and received by $2.4 \mathrm{GHz}$ radio transceiver and base-band. According to the user's navigation it switches the relay pin as set in the code and turns two motor on for the forward direction and for the left directed movement the specific pin of relay turns on and left motor will be turned on and right motor will be turned off. The opposite scenario happens for right directed path movement.

For displaying the voice command via $16 \times 2$ matrix and 5 $\mathrm{x} 8$ dots with cursor, converted text are showed according to the direction of Built-in controller. It requires $\quad+5 \mathrm{~V}$ or $+3 \mathrm{~V}$ power supply. The $1 / 16$ duty cycle and $\mathrm{B} / \mathrm{L}$ to be driven by pin 1 , pin 2 or pin 15, pin 16. Again coded arduino uno module transmits and receives the voice both via Bluetooth module.

It offers excellent non-contact range detection with high accuracy and stable readings in an easy-to-use package. The ultrasonic sensor (SONAR) detects the obstacles from $2 \mathrm{~cm}$ to $400 \mathrm{~cm}$ or 1 " to 13 feet with the mechanism of sonar detection. Its operation is not affected by sunlight or black material. The requirement of 2 pins of the Arduino's digital pins was connected so far. The Effectual Angle $<15^{\circ}$ and measuring $30^{\circ}$ with sensing the pulse with frequency sending (TRIG), receiving $(\mathrm{ECHO})$ time are operated by $+5 \mathrm{~V}$. With the time, the distance of the object is observed. If no object has been detected, the pulses are not received back, so the echo pin returns no pulse or a 0 .

Atmega32 was programmed by Serial peripheral interface or by Parallel programming. 32 Programmable I/O and Lines 40-pin PDIP lines, 44-lead TQFP, and 44-pad MLF were used through the burned ATmega32 microcontroller based circuit which is operated by an external $4.5 \mathrm{~V}$ operated voltage. The coded micro controller get the directions and activate the IR sensor to motivate the sensing light ray for the surface in front of the wheelchair which will be received by the photodiode and make a sense with strong signal if and only if it finds no surface as well as any hole or crack.

As soon as the digital pulse High voltage is triggered for detecting the heat from blood of living being, a buzzer module sense through some time being. It helps to be informed about get rid of accidental issues from the front side of the chair for the user who cannot see. The voltage is low in the case of no motion detected. Its range is up to 20 feet ( 6 meters) as well as $110^{\circ} \times 70^{\circ}$ detection range.

Home automation is powered by coded arduino uno $+5 \mathrm{~V}$. For the home automation procedure a coded arduino uno has been used where relay pin is connected with the arduino and load as well. This process goes to $+5 \mathrm{~V}$ from the
USB of arduino. By the direction of coded arduino the ANDROID application sends the signal and receives the signal via Bluetooth module. Thus the load can be controlled so far.

\subsection{Pseudo Code}

Accumulated code of Android control wheelchair and home automation has been described below:

Firstly

Define pin 2 for Relay 1

Define pin 3 for Relay 2

Define pin 4 for Relay 3

Define pin 5 for Relay 4

Define Pin 1 for TX in Bluetooth Module

Define Pin 0 for RX in Bluetooth Module

Serial begins $9600 \mathrm{MHz}$ for Bluetooth Module

If

State $=\mathrm{A}$

Relay $1=\mathrm{ON}$

Light will be ON

Else if

State $=\mathrm{a}$

Relay $1=$ Off

Light will be off

Else if

State $=\mathrm{B}$

Relay $2=\mathrm{ON}$

Fan will be ON

Else if

State $=\mathrm{b}$

Relay $2=$ Off

Fan will be off

Else if

State $=1$

Relay $3=\mathrm{ON}$ and Relay $4=\mathrm{ON}$

Wheelchair will go Forward direction

Else if

State $=2$

Relay $3=$ Off and Relay $4=$ On

Wheelchair will turn left direction

Else if

State $=3$

Relay $3=$ Off and Relay $4=$ Off

Wheelchair will stop

Else

State $=4$

Relay $3=$ On and Relay $4=$ Off

Wheelchair will turn right direction

Now the code of obstacle Sensing part is written below:

Firstly

Define Pin 11 for RS in LCD

Define Pin 10 for EN in LCD

Define Pin 5 for D4 in LCD

Define Pin 4 for D5 in LCD

Define Pin 3 for D6 in LCD 
Define Pin 2 for D7 in LCD

Define Pin 8 for Trig in sonar

Define Pin 9 for Echo in sonar If

Trig pin $=$ Low and Echo pin $=$ High

Calculate distance in $\mathrm{Cm}=$ duration*0.034/2

Calculate distance in Inch $=$ duration $* 0.0133 / 2$

Show obstacle distance in LCD display delay $10 \mathrm{~ms}$.

\subsection{Accumulated Code of Voice Commanded LCD Display and PIR}

The used code for accumulation of voice commands are described below:

The used code for accumulation of voice commands are described below:

Firstly

Define pin 9 for PIR input

Define Pin 10 for Buzzer

Define Pin 12 for RS in LCD

Define Pin 11 for EN in LCD

Define Pin 5 for D4 in LCD
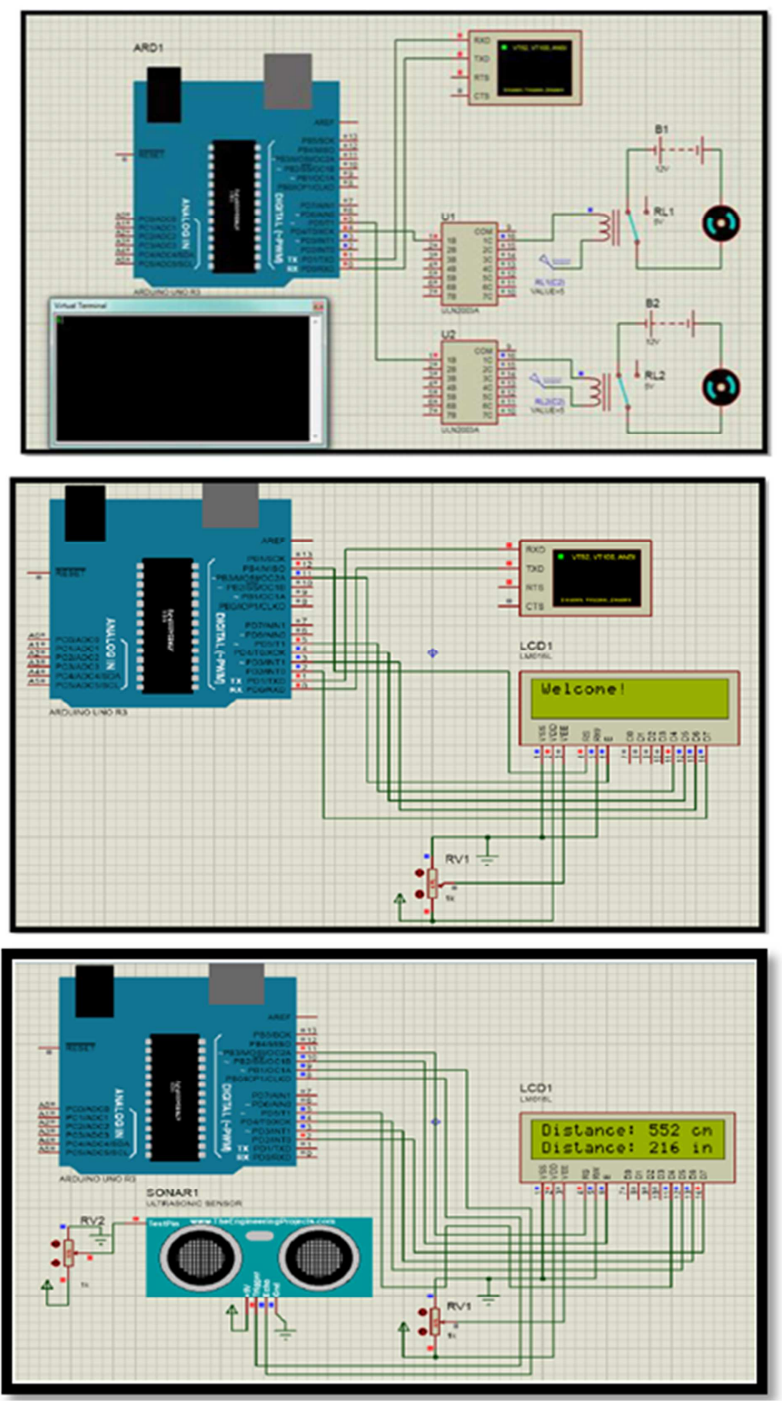

Figure 4. Individual circuit diagrams of Android control wheelchair movement (top left), Android control home automation (top right), Android control voice

command display (middle left), living being sensing (middle right), obstacle sensing (bottom left) and crack detection respectively (Bottom right).
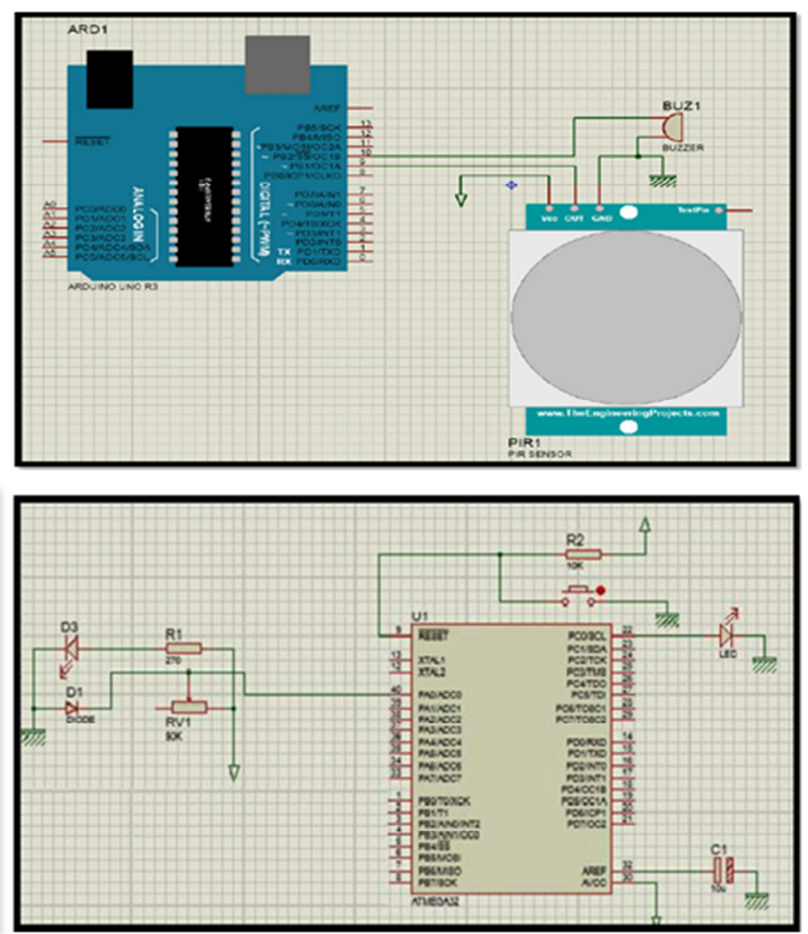

Define Pin 4 for D5 in LCD

Define Pin 3 for D6 in LCD

Define Pin 2 for D7 in LCD

Define Pin 1 for TX in Bluetooth Module

Define Pin 0 for RX in Bluetooth Module

Serial begin $9600 \mathrm{MHz}$ for Bluetooth Module

Pin $9=$ High, Buzzer will be ON

Delay $150 \mathrm{~ms}$

Else if

Pin $9=$ Low, Buzzer will be OFF

Else if

Pin 0 for RX in Bluetooth Module receive any message

LCD display show the message

Else

LCD display will be blank

\section{Individual Circuit Diagram}

Each individual circuit diagrams can clarify the operation done by the wheelchair.

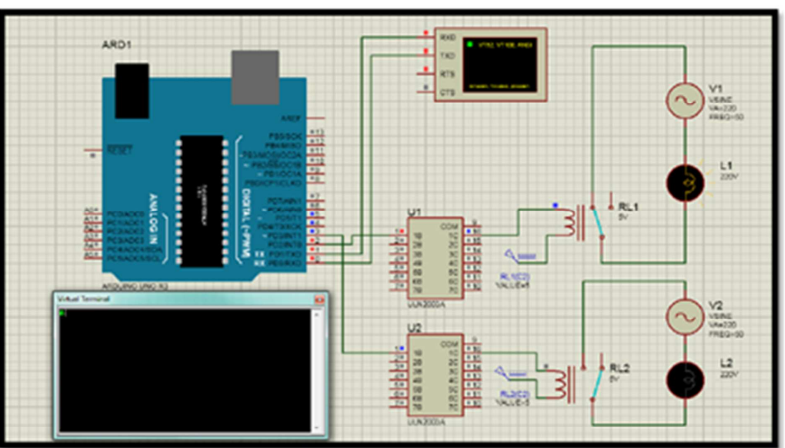



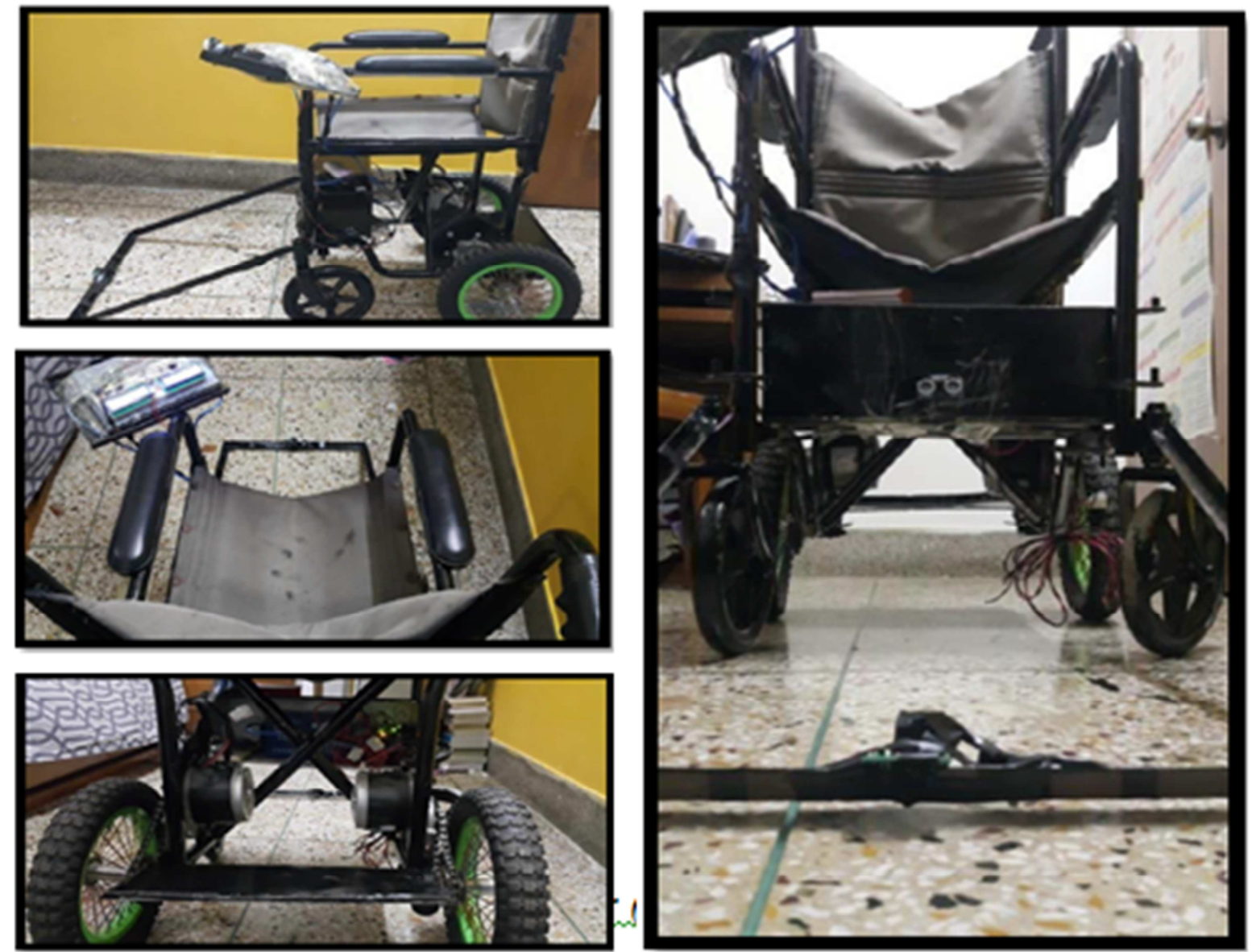

Figure 5. Intelligent Wheelchair (Side, Top, Back and Front view).

Hardware connections and outcomes as well as Individual outcomes has discussed on the following paragraphs.

\section{Android Control Wheelchair Movement and Home Automation}

Now, it can be called like a smart-phone compatible device as it can be controlled by any android system's phone.
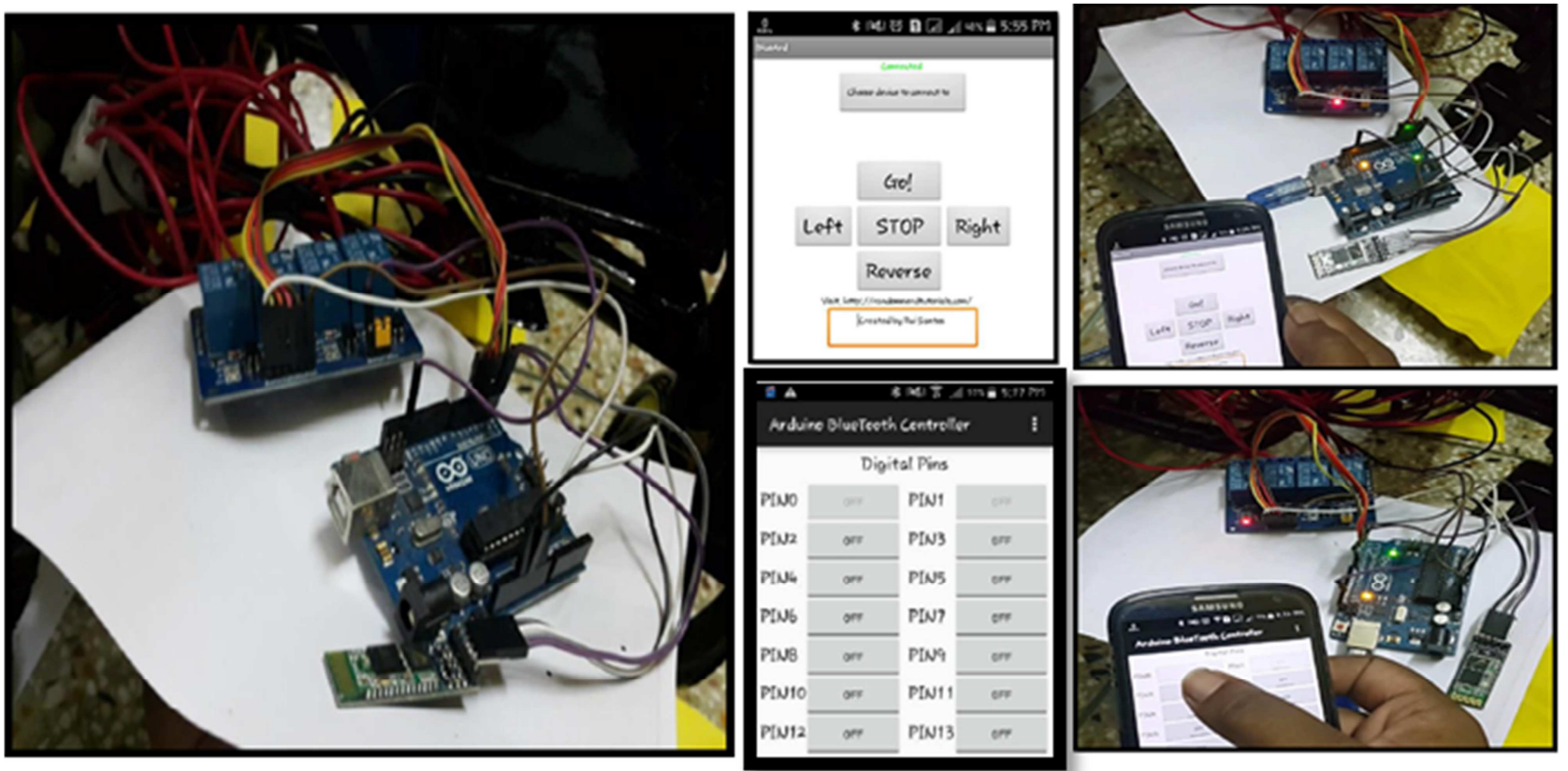

Figure 6. Using android software relay pin as well as movement of wheelchair and home appliances control. 


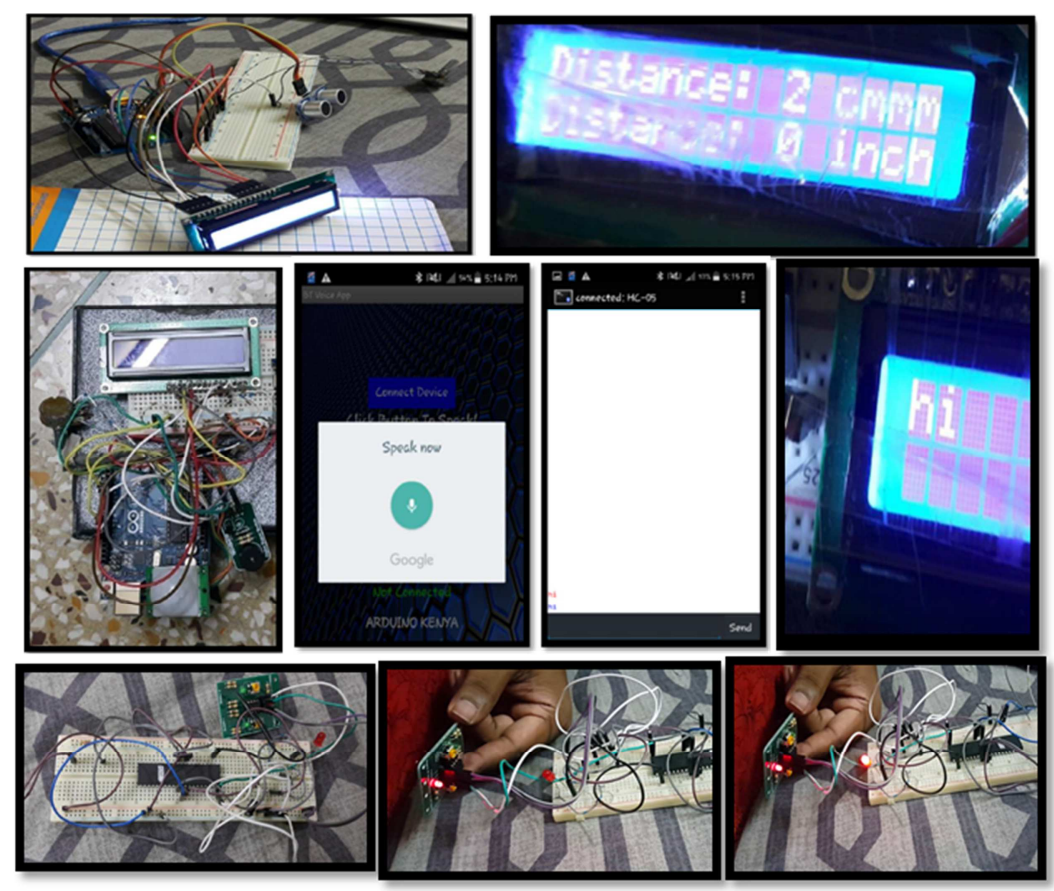

Figure 7. The circuit connection of sonar and obstacle sensing via LCD display (row 1), The circuit connection and using mobile directed voice showed in LCD display (row 2), Circuit connection of crack sensing and signalling through LED while no hole/crack found (row 3).

\section{Approximate Cost}

The estimation and expenses for the necessary equipment is mentioned below:

Table 1. Cost of constructing the wheelchair.

\begin{tabular}{llll}
\hline No. & Material Name & Quantity & Price (In BDT) \\
\hline 1 & Wheelchair & 1 & 2000 \\
2 & Motor 12V & 2 & 5000 \\
3 & Arduino Uno & 3 & 600 \\
4 & Atmega32 & 1 & 200 \\
5 & LCD display & 2 & 400 \\
6 & Bluetooth Module & 2 & 1000 \\
7 & Relay Module & 1 & 250 \\
8 & Buzzer Module & 1 & 30 \\
9 & Sonar & 1 & 150 \\
10 & IR & 1 & 150 \\
11 & PIR & 1 & 150 \\
12 & Battery 12V & 1 & 3000 \\
13 & Bread Board & 2 & 360 \\
14 & Registers & 5 & 10 \\
15 & LED and Jumper Wire & 1 & 200 \\
Total & & & 13500 \\
\hline
\end{tabular}

\section{Advantage over Traditional Wheelchair}

This wheel chair does not require hand motivated motion. Rather by using an android smart phone with specific application, this wheelchair can be moved easily in the desired direction Independence can be felt which is almost similar to walk. Handicapped people are quite sufferer to make a communication with others. Especially for the people with auditory disability, likewise the targeted goal was achieved by making the wheelchair with an Android control software based operation system where the directions, messages or voice can be seen by the user easily. User can also use this option for communication. However, this chair has identification mechanism for the safety of handicapped people. The sensing system of distance about the obstacle, identification of the living beings and detection of crack on the surface provide more safety. This wheel chair provides the home automation system as an extra ordinary function indeed. For enjoying the outdoors, one can enjoy picnics with friends at the park, explore park trails, bird watch, shopping at favourite stores, dine at restaurants, travel, visit friends and family and much more. Wheelchairs provide the user with a more active lifestyle that usually results in a happier, healthier more positive outlook on life.

\section{Conclusion}

This bright and innovative design will help for the handicapped person along with reducing the sophisticated life style of those types of patient. The principal job will be too helpful for the users so far for the revolution obviously this chair has to be further developed and manufactured. The world will see the continuous invention with pioneering Excellences. For higher strength of the body composite materials can be used. An automation robotic system with the help of artificial intelligence can be used to make a helper for the handicap person.

\section{Future Work Plans}

The future robotic wheelchair can be developed as it can learn the layout of its environment (hospital, rehabilitation 
centre, home, etc.) through a directed tour given by the user or the user's caregivers. Subsequently, the wheelchair can move to any previously-named location under voice command (e.g., "Take me to the cafeteria"). This technology is appropriate for people who have lost mobility due to brain injury or the loss of limbs, but who retain speech and using GPS system. The technology can be enhanced with Tongue Motion Driver to move the chair by the movement of tongue which will be easier for totally paradise people. It can be modified by gesture technology or voice commanded technology. The technology can also enhance safety for users modified by caterpillar tracks which can be used through stairs. Again for the movement in outside this wheelchair can be built up with the operation of GSM for perfect communication of the user with others. Moreover, a photovoltaic thin film solar cell can be fabricated because it is cheap and the energy consumption will be reduced.

\section{Acknowledgements}

The research team would like to express the greatest gratitude to Tajbia Karim and Dr. Mohammad Nasir Uddin of the Department of Electrical and Electronic Engineering, American International University-Bangladesh.

\section{References}

[1] Lisbeth Nilsson, Universal Design Of A Powered Wheelchair For People With Cognitive Disabilities, Journal of Lund University, Aug 2011.

[2] Dejan Nedelkovski, Ultrasonic Sensor HC-SR04 and Arduino Tutorial, Journal of Mechatronics, Jan 2014.

[3] Hasan U. Zaman, Md. Majidul Haque Bhuiyan, Montashir Ahmed, S. M Tarek Aziz, A novel design of line following robot with multifarious function ability, International Conference on Microelectronics, Computing and Communications (MicroCom), Jan 2016.

[4] Brígida Mónica Faria, Luís Paulo Reis, Nuno Lau, A Survey on Intelligent Wheelchair Prototypes and Simulators, Part of the Advances in Intelligent Systems and Computing book series, Mar 2014

[5] Arun Kumar Garg, Edge Detector Robot with ATmega32 using Analog IR Sensor, Journal of IR Sensors, Jun 2014.

[6] Tazdik Patwary Plateau, Evaluation of Tensile Strength of Jute Fiber Reinforced Polypropylene Composite, Journal of Advances in Materials, Science PG, Dec 2017.

[7] Timothy P. Sabol, Evelyn S. Haley, Wheelchair Evaluation for the Older Adult, Journal of Clinics in Geriatric Medicine, Elsevier, May 2006.

[8] Susan I. Fuhrman, Patricia E. Karg, Gina E. Bertocci, Effect of wheelchair headrest use on pediatric head and neck injury risk outcomes during rear impact, Journal of Accident Analysis \& Prevention, Elsevier, July 2008.

[9] A. Naga Rajesh, S. Chandralingam, T. Anjaneyulu, K. Satyanarayana, EOG Controlled Motorized Wheelchair for Disabled Persons, International Journal of Mechanical and
Materials Engineering, May 2015.

[10] Tazdik Patwary Plateau, Md. Majidul Haque Bhuiyan, A heuristic proposition of efficient copper-electrodeposited ptype thin film for CZTS solar cell, International Conference on Electrical, Computer and Communication Engineering (ECCE), Feb 2017.

[11] Lisa Hilliard, Phillip Dunston, James Mc Glothlin, Bradley S. Duerstock, Designing Beyond the ADA - Creating an Accessible Research Laboratory for Students and Scientists with Physical Disabilities, Journal of Chemical Education, Nov 2012.

[12] H. Sermeno-Villalta, J. Spletzer, Vision-based control of a smart wheelchair for the automated transport and retrieval system (ATRS), IEEE International Conference on Robotics and Automation, ICRA, May 2006.

[13] E. K. Skordilis, C. Sherrill, A. Yilla D. Koutsouki, N. A. Stavrou, Use of the Sport Orientation Questionnaire with Wheelchair Athletes: Examination of Evidence for Validity, Perceptual and Motor Skills, August 2002.

[14] Tazdik Patwary Plateau, A Cheap Way to Develop Absorber Layer of Solar Cell Using $\mathrm{CuO}$ Thin Film, 4th International Conference on Advances in Electrical Engineering (ICAEE), IUB Campus, Dhaka, Bangladesh, Sept 2017.

[15] Chandra Foreman, Jennifer Hardin, The Challenges Of Wheelchair Securement: Searching For Solutions, Journal of Urban Transportation, Feb 2001.

[16] Ruwaidy Mat Rasul, Standardized Wheelchair Assessment Towards Practical Wheelchair Design, Journal of Advanced Nursing, Oct 2013.

[17] A. F. Kadmin, A. Z. Jidin, Abu Bakar, K. A. A Aziz, W. N. Abd Rashid, Wireless Voice-Based Wheelchair Controller System, Journal of Telecommunication, Electronic and Computer Engineering, Apr 2007.

[18] Mark Hartridge, Barry R. Seeger, International Wheelchair Standards: A Study of Costs and Benefits, The Official Journal of RESNA- Assistive Technology, Oct 2010.

[19] Amos G. Winter, Mario A. Bollini, Benjamin M Judge, Natasha K Scolnik, Harrison F O'Hanley, Daniel S Dorsch, Sudipto Mukherjee, Daniel D Frey, Stakeholder-Driven Design Evolution of the Leveraged Freedom Chair Developing World Wheelchair, American Society of Mechanical Engineers (ASME), November 2012.

[20] E. B. Vander Poorten, E. Demeester, E. Reekmans, J. Philips, A. Hüntemann, J. De Schutter, Powered wheelchair navigation assistance through kinematically correct environmental haptic feedback, May 2012.

[21] Robin L. Drespel, An Evaluation of Wheelchair Restraint Systems Used in the Transportation of Disabled Persons, Retrospective Theses and Dissertations, Dec 1985.

[22] AndrewDavies, NicolaChristie, An exploratory study of the experiences of wheelchair users as aircraft passengers implications for policy and practice, Journal of IATSS Research, July 2017.

[23] Jyothi Rani, B. Adhithya, Eye Controlled Wheelchair System Along With Hand Talk, International Journal of Engineering, Technology, Management and Research, Nov 2016. 